\begin{tabular}{|c|l|}
\hline Title & Uniform global solvability of the rotating Navier-Stokes equations for nondecaying initial data \\
\hline Author(s) & Giga, Y oshikazu; Inui, Katsuya; Mahalov, A lex; Saal, Jurgen \\
\hline Citation & Hokkaido University Preprint Series in Mathematics, 924, 1-17 \\
\hline Issue Date & 2008-09 \\
\hline DOI & 10.14943/84073 \\
\hline Doc URL & http://hdl.handle.net/2115/69731 \\
\hline Type & bulletin (article) \\
\hline File Information & pre924.pdf \\
\hline
\end{tabular}

Instructions for use 


\title{
Uniform global solvability of the rotating Navier-Stokes equations for nondecaying initial data
}

\author{
Yoshikazu Giga $^{1}$, Katsuya Inui ${ }^{2,3}$, Alex Mahalov ${ }^{4}$, and Jürgen Saal ${ }^{5}$
}

Dedicated to Professor Ciprian Foias on the occasion of his seventy-fifth birthday

${ }^{1}$ Graduate School of Mathematics Sciences, University of Tokyo, 3-8-1 Komaba, Meguro-ku, Tokyo, 153-8914 Japan, Facsimile (81)-3-5465-7023, labgiga@ms.u-tokyo.ac.jp

${ }^{2}$ Visiting Researcher Department of Electronic and Computer Engineering, Musashi Institute of Technology

1-28-1 Tamazutsumi, Setagaya, Tokyo, 158-8557 Japan E-mail: pt125456@kanagawa-u.ac.jp

${ }^{3}$ Part-time Teacher Faculty of Engineering, Saitama Institute of Technology, Faculty of Science, Kanagawa University, Faculty of Engineering, University of Yamanashi

${ }^{4}$ Department of Mathematics and Statistics, Arizona State University, Tempe, AZ 85287-1804, USA, Facsimile (1)-480-357-9791, mahalov@asu.edu

${ }^{5}$ University of Konstanz, Department of Mathematics, Box D 187, 78457 Konstanz, Germany, juergen.saal@uni-konstanz.de

\begin{abstract}
We establish a global existence result for the rotating Navier-Stokes equations with nondecaying initial data in a critical space which includes a large class of almost periodic functions. The scaling invariant function space we introduce is given as the divergence of the space of $3 \times 3$ fields of Fourier transformed finite Radon measures. The smallness condition on initial data for global existence is explicitly given in terms of the Reynolds number. The condition is independent of the size of the angular velocity of rotation.
\end{abstract}

Mathematical Subject Classification (2000). Primary: 76D05, 76U05, Secondary: 35Q30, 28B05, 28C05. 
Keywords. Navier-Stokes equations, Coriolis force, global solutions, Radon measures, almost periodic initial data

\section{Introduction and main results}

We study the incompressible rotating Navier-Stokes equations on $\mathbb{R}^{n} \times(0, \infty)$ :

$$
\left\{\begin{aligned}
\partial_{t} u-\nu \Delta u+\Omega J u+(u \cdot \nabla) u & =-\nabla p & & \text { in } \mathbb{R}^{n} \times(0, \infty), \\
\operatorname{div} u & =0 & & \text { in } \mathbb{R}^{n} \times(0, \infty), \\
\left.u\right|_{t=0} & =u_{0} & & \text { in } \mathbb{R}^{n}
\end{aligned}\right.
$$

where $u(x, t)$ is the velocity vector field and $p$ is the pressure. Here $\nu$ is the kinematic viscosity coefficient. For the moment we explain the three dimensional case $(n=3)$, which is the main focus of this paper. In $\mathbb{R}^{3}$, the skew-symmetric term $J u=\Omega \mathbf{e}_{3} \times u$ represents the Coriolis force, where $\Omega$ denotes twice the angular velocity of rotation. The coordinate system is chosen in a way such that the rotation is around the axis $\mathbf{e}_{3}=(0,0,1)^{T}$.

The main objectives of the present work are: (i) to prove the existence of global solutions for system (1.1) for a class of spatially nondecaying initial data which includes a large class of almost periodic funtions; (ii) to quantify the smallness condition and to prove that the bound for the solution is independent of the angular velocity $\Omega$. This has some relevance for several reasons: existence of solutions with norms uniformly bounded in $\Omega$ in spaces including functions nondecaying at infinity are essential in studies of statistical properties of turbulence, see e.g. [8], [17, 18], and in the analysis of fast oscillating singular limits for system (1.1), see [1], [2] and [15].

In our previous work [10] and [12], we studied the system (1.1) in the new function space $\mathrm{FM}_{0}\left(\mathbb{R}^{3}, \mathbb{C}^{3}\right)$, which represents the space of finite Fourier transformed Radon measures with no point mass at the origin. Note that this space is different from the space of pseudo measures which is the space of Fourier transformed bounded functions as introduced by [16] to their study of the Navier-Stokes equations; see also [3].

In [10] local-in-time existence is proved whereas in [12] we show global existence for small Reynolds number under the additional condition that the Fourier transform of the initial data is supported in a sum-closed frequency set. The crucial point is that all results and conditions in [10] and [12] are uniformly in the Coriolis parameter $\Omega$ and that the space contains nondecaying functions including a class of almost periodic functions.

In the present paper we will prove uniform global in time solvability of (1.1) for 
small initial data in the critical space

$$
\mathrm{FM}_{0}^{-1}\left(\mathbb{R}^{3}, \mathbb{C}^{3}\right):=\operatorname{div} \mathrm{FM}_{0}\left(\mathbb{R}^{3}, \mathbb{C}^{3}\right)^{3} .
$$

The fact that we work with a space of Fourier transformed quantities makes our approach elementary and clear, see also [10] and [12]. In fact, as an interesting outcome, similarly to [12], we will obtain an explicit representation of the smallness condition in terms of the Reynolds number, see Theorem 1.1. We also emphasize that all results given here include the case $\Omega=0$, i.e., the standard Navier-Stokes equations. The results presented in this paper improve the results given in [12] in the sense that we can remove the additional condition on the support of the Fourier transformed initial data. This has the advantage that our new results hold for arbitrary almost periodic functions as initial data, whereas the results in [12] merely admit a restricted class of almost periodic functions.

Our paper provides the first global result for the rotating Navier-Stokes equations with general small initial data which are spatially nondecaying. Even without the Coriolis term the Navier-Stokes equations with spatially nondecaying initial data is less studied in the literature compared with the case of decaying initial data. There is a nice review paper [3] and a introductory book [5] on well-posedness for decaying initial data for the Navier-Stokes equations without the Coriolis therm i.e. $\Omega=0$. For two dimensional case global existence for arbitrary bounded initial data is known [13] (for local existence, see e.g. [9]). A celebrated result for the standard Navier-Stokes equations $(\Omega=0)$ in the critical space $\mathrm{BMO}^{-1}\left(\mathbb{R}^{n}\right)$ is obtained in [14]. This is the only global existence result for small initial data which may not decay at the spatial infinity except periodic initial data.

As $\mathrm{BMO}^{-1}\left(\mathbb{R}^{n}\right)$, the space $\mathrm{FM}^{-1}\left(\mathbb{R}^{n}\right)$ is also a critical space for the NavierStokes equations, i.e., it is scaling invariant under the transformation

$$
u(x, t) \mapsto \alpha u\left(\alpha x, \alpha^{2} t\right),
$$

with the corresponding scaling for the pressure. Furthermore, also in the space $\mathrm{FM}^{-1}\left(\mathbb{R}^{n}\right)$ the initial value problem is borderline ill-posed in the sense that the proof of local well-posedness by the usual fixed point argument is supposed to fail in $\mathrm{FM}^{-1-\varepsilon}\left(\mathbb{R}^{n}\right)$ for $\varepsilon>0$. By the fact that

$$
\mathrm{FM}\left(\mathbb{R}^{n}\right) \subseteq \mathrm{BUC}\left(\mathbb{R}^{n}\right) \subseteq \mathrm{BMO}\left(\mathbb{R}^{n}\right)
$$

it follows that (see [14] and Lemma 2.9(i))

$$
\mathrm{FM}^{-1}\left(\mathbb{R}^{n}\right)=\operatorname{div} \mathrm{FM}\left(\mathbb{R}^{n}\right)^{n} \subseteq \operatorname{div} \mathrm{BMO}\left(\mathbb{R}^{n}\right)^{n}=\mathrm{BMO}^{-1}\left(\mathbb{R}^{n}\right),
$$

i.e., our class is contained in $\mathrm{BMO}^{-1}\left(\mathbb{R}^{n}\right)$. However, we emphasize that the main intention of this paper is not to find the largest class of well-posedness, but to derive 
global well-posedness uniformly in the Coriolis parameter $\Omega$ without the restrictive assumption on the initial data imposed in [12]. Observe that in $\mathrm{BMO}^{-1}\left(\mathbb{R}^{n}\right)$ the uniformness in $\Omega$ is expected to fail, by the fact that a multiplier result as Proposition 2.5 is not available in that space. In $\mathrm{BMO}^{-1}\left(\mathbb{R}^{n}\right)$ polynomial growth in $\Omega$ for the Poincaré-Riesz semigroup $\mathrm{e}^{\Omega P J P t}$ arising from the Coriolis term is expected. This behavior is also expected for other known spaces containing nondecaying functions as the homogeneous Besov space $\dot{B}_{\infty, 0}^{-1}\left(\mathbb{R}^{n}\right)$, whereas in the space of uniformly continuous functions $\mathrm{BUC}^{-1}\left(\mathbb{R}^{n}\right)$, for instance, the Poincaré-Riesz semigroup doesn't even exist. We note that the authors of [7] developed new techniques allowing to prove growth in $\Omega$ of the Poincaré-Riesz semigroup in certain functional spaces.

The global existence for large $|\Omega|$ is known for periodic initial data ([1], [2]) and for bounded cylindrical domains with slip boundary conditions on vertical plates $([12])$. The global existence for small initial data whose smallness is uniform in $\Omega$ is known for periodic initial data ([1], [2]). For results on global well-posedness for (1.1) the reader is referred to [4]. In this paper we present the first available global existence results for small real-valued almost periodic initial data. In [11] we considered Navier-Stokes equation with complex almost periodic initial data but no real-valued data was allowed.

We formulate our main results in the $n$-dimensional setting, i.e., $J \in \mathbb{R}^{n \times n}$ is a suitable skew-symmetric matrix (see (2.1)) such that $J u=\mathbf{e}_{3} \times u$, if $n=3$. We consider (1.1) in the space

$$
\mathrm{FM}_{0, \sigma}\left(\mathbb{R}^{n}, \mathbb{C}^{n}\right):=\left\{\widehat{u}: u \in \mathrm{M}\left(\mathbb{R}^{n}, \mathbb{C}^{n}\right), \operatorname{div} u=0, u(\{0\})=0\right\} .
$$

Here $\mathrm{M}\left(\mathbb{R}^{n}, \mathbb{C}^{n}\right)$ denotes the space of finite $\mathbb{C}^{n}$-valued Radon measures (see the next section for a precise definition), which can be regarded as the dual space of

$$
C_{\infty}\left(\mathbb{R}^{n}, \mathbb{C}^{n}\right)=\left\{u \in C\left(\mathbb{R}^{n}, \mathbb{C}^{n}\right): \lim _{R \rightarrow \infty}\|u\|_{L^{\infty}\left(\mathbb{R}^{n} \backslash B_{R}\right)}=0\right\} .
$$

Here $B_{R}$ denotes the ball with center 0 and radius $R$ and $\mathbb{R}^{n}$ and $\mathbb{C}^{n}$ are always equipped with the Euclidean norm Initial data will be an element of

$$
\mathrm{FM}_{0, \sigma}^{-1}\left(\mathbb{R}^{n}, \mathbb{C}^{n}\right):=\operatorname{div} \mathrm{FM}_{0, \sigma}\left(\mathbb{R}^{n}, \mathbb{C}^{n}\right)^{n} .
$$

The spaces $\mathrm{FM}_{0, \sigma}\left(\mathbb{R}^{n}, \mathbb{C}^{n}\right)$ and $\mathrm{FM}_{0, \sigma}^{-1}\left(\mathbb{R}^{n}, \mathbb{C}^{n}\right)$ are equipped with their canonical norms. Our main results read as follows.

Theorem 1.1. (unique existence of global mild solution) Let $\nu>0, \Omega \in \mathbb{R}$, and $u_{0} \in \mathrm{FM}_{0, \sigma}^{-1}\left(\mathbb{R}^{n}, \mathbb{C}^{n}\right)$. Then, if

$$
\left\|u_{0}\right\|_{\mathrm{FM}_{0, \sigma}^{-1}}<\frac{\nu(2 \pi)^{n / 2}}{2 \sqrt{n}},
$$


there exists a unique global mild solution $u \in L^{2}\left((0, \infty), \mathrm{FM}_{0, \sigma}\left(\mathbb{R}^{n}, \mathbb{C}^{n}\right)\right)$ of the Navier-Stokes equations (1.1).

Note that the space $\mathrm{FM}_{0, \sigma}^{-1}\left(\mathbb{R}^{n}, \mathbb{C}^{n}\right)$ contains (complex-valued or pure real-valued) almost periodic functions of the form

$$
u_{0}(x)=\sum_{j=1}^{\infty} a_{j} e^{i \lambda_{j} \cdot x}, \quad x \in \mathbb{R}^{n}, a_{j} \in \mathbb{C}^{n}, \lambda_{j} \in \mathbb{R}^{n} \backslash\{0\},
$$

whenever $\sum_{j=1}^{\infty}\left(\max _{i}\left|a_{j i}\right|\right) /\left(\min _{i}\left|\lambda_{j i}\right|\right)<\infty$ and $\operatorname{div} u_{0}=0$.

Remark 1.2. The smallness condition (1.2) is equivalent to $R e<\frac{(2 \pi)^{n / 2}}{2 \sqrt{n}}$, where $R e:=\left\|u_{0}\right\|_{\mathrm{FM}_{0, \sigma}^{-1}} / \nu$ is the Reynolds number. We note that the norm $\left\|u_{0}\right\|_{\mathrm{FM}_{0, \sigma}^{-1}}$ has the physical units of (velocity $x$ length).

For the obtained mild solution we have

Theorem 1.3. (regularity of mild solutions) Assume that $u_{0} \in \mathrm{FM}_{0, \sigma}^{-1}\left(\mathbb{R}^{n}, \mathbb{C}^{n}\right)$ satisfies (1.2). Let $u \in L^{2}\left((0, \infty), \mathrm{FM}_{0, \sigma}\left(\mathbb{R}^{n}, \mathbb{C}^{n}\right)\right)$ be the solution obtained in the above theorem. In addition, assume that $u_{0} \in \mathrm{FM}_{0, \sigma}\left(\mathbb{R}^{n}, \mathbb{C}^{n}\right)$. Then, we have

(1) $\nabla u(t) \in L^{2}\left([0, T], \operatorname{FM}\left(\mathbb{R}^{n}, \mathbb{C}^{n}\right)\right)(0<T \leq \infty)$, and

$$
\|\nabla u\|_{L^{2}((0, T), \mathrm{FM})} \leq \frac{1}{\sqrt{2 \nu}}\left\|u_{0}\right\|_{\mathrm{FM}} \quad(0<T \leq \infty) .
$$

(2) Moreover, we have

$$
\begin{aligned}
& t^{1 / 2} \nabla u(t) \in C\left(\left[0, T_{0}\right], \operatorname{FM}\left(\mathbb{R}^{n}, \mathbb{C}^{n}\right)\right) \quad \text { for } 0<T_{0}(<\infty), \\
& \lim _{t \rightarrow 0} t^{1 / 2}\|\nabla u(t)\|_{\mathrm{FM}}=0 .
\end{aligned}
$$

In the case $n=3$, we also have

Theorem 1.4. (local classical solution) Assume that $u_{0} \in \mathrm{FM}_{0, \sigma}^{-1}\left(\mathbb{R}^{3}, \mathbb{R}^{3}\right) \cap$ $\mathrm{FM}_{0, \sigma}\left(\mathbb{R}^{3}, \mathbb{R}^{3}\right)$ satisfies (1.2). Let $u(t)$ be the mild solution obtained in the above theorems in the case $n=3$. If we set

$$
\partial_{i} p(t)=\partial_{i} \sum_{j, k=1}^{3} R_{j} R_{k} u^{j} u^{k}(t)+\Omega R_{i}\left(R_{2} u^{1}-R_{1} u^{2}\right)(t) \quad \text { for } t>0, \quad i=1,2,3 .
$$

then, the pair $(u, \nabla p)$ solves (1.1) for $\mathbb{R}^{3} \times\left(0, T_{0}\right)$ with any $T_{0}<\infty$. 


\section{Radon measures and key estimates}

We introduce some notations. By $\mathscr{L}(X)$ we denote the space of all bounded operators on the Banach space $X$. As mentioned before, the spaces $\mathbb{R}^{n}$ and $\mathbb{C}^{n}$ are always equipped with the Euclidean norm. For $G \subseteq \mathbb{R}^{n}, C(G, X)$ denotes the space of continuous functions and $\operatorname{BC}(G, X)$ the space of all bounded and continuous functions on $G$ with values in $X$. In this note we denote the Fourier transform by

$$
\widehat{u}(\xi)=\mathcal{F} u(\xi):=\frac{1}{(2 \pi)^{n / 2}} \int_{\mathbb{R}^{n}} e^{-i \xi \cdot x} u(x) \mathrm{d} x, \quad u \in \mathcal{S}\left(\mathbb{R}^{n}\right),
$$

where $\mathcal{S}\left(\mathbb{R}^{n}\right)$ denotes the Schwartz space of rapidly decreasing functions. Its extension on $\mathcal{S}^{\prime}\left(\mathbb{R}^{n}\right)$ is defined as usual.

We recall briefly some properties of the spaces $\mathrm{FM}$ and $\mathrm{FM}_{0}$ from [10] and [12]. For a comprehensive introduction to vector measures we refer to [6].

Definition 2.1. Let $\mathscr{A}$ be a $\sigma$-algebra over $\mathbb{R}^{n}$. The set map $\mu: \mathscr{A} \rightarrow \mathbb{C}^{n}$ is called a finite $\mathbb{C}^{n}$-valued (or more general vector valued) Radon measure, if

(i) $\mu$ is a $\mathbb{C}^{n}$-valued measure, i.e., it is $\sigma$-additive and $\mu(\emptyset)=0$,

(ii) the variation of $\mu$ given by

$$
|\mu|(\mathcal{O}):=\sup \left\{\sum_{E \in \Pi(\mathcal{O})}|\mu(E)|: \Pi(\mathcal{O}) \subseteq \mathscr{A} \text { finite decomposition of } \mathcal{O}\right\}
$$

for $\mathcal{O} \in \mathscr{A}$ is a finite Radon measure. (Note that $\Pi(\mathcal{O}) \subseteq \mathscr{A}$ is a decomposition of $\mathcal{O} \in \mathscr{A}$, if $A \cap B=\emptyset$ for all $A, B \in \Pi$ with $A \neq B$ and $\bigcup_{A \in \Pi} A=\mathcal{O}$.)

We denote by $\mathrm{M}\left(\mathbb{R}^{n}, \mathbb{C}^{n}\right)$ the space of all finite $\mathbb{C}^{n}$-valued Radon measures.

Recall that $\eta: \mathscr{A} \rightarrow[0, \infty)$ is a finite Radon measure, if $\eta\left(\mathbb{R}^{n}\right)<\infty$ and if it is Borel regular, that is, if $\mathscr{B} \subseteq \mathscr{A}$ and if for each $A \subseteq \mathbb{R}^{n}$ there exists a $B \in \mathscr{B}$ such that $\eta^{*}(A)=\eta^{*}(B)$, where $\mathscr{B}$ denotes the Borel $\sigma$-algebra over $\mathbb{R}^{n}$ and $\eta^{*}$ denotes the outer measure associated to $\eta$. Also observe that we indentify $\eta$ by its outer measure, so that $\eta$ is complete in the sense that all subsets $B$ of a set $A \in \mathscr{A}$ satisfying $\eta(A)=0$ belong to $\mathscr{A}$. By the Riesz representation theorem it is well-known that $\mathrm{M}\left(\mathbb{R}^{n}, \mathbb{C}\right)$ can be regarded as the dual space of

$$
C_{\infty}\left(\mathbb{R}^{n}, \mathbb{C}\right)=\left\{u \in C\left(\mathbb{R}^{n}, \mathbb{C}\right): \lim _{R \rightarrow \infty}\|u\|_{L^{\infty}\left(\mathbb{R}^{n} \backslash B_{R}\right)}=0\right\},
$$

where $B_{R}$ denotes the ball with center 0 and radius $R$ (see [6]). 
Remark 2.2. (a) It can be shown that $\mu$ is a finite $\mathbb{C}^{n}$-valued measure if and only if the variation $|\mu|$ is a finite nonnegative measure.

(b) Equipped with the norm $\|\mu\|_{\mathrm{M}}:=\|\mu\|_{\mathrm{M}\left(\mathbb{R}^{n}, \mathbb{C}^{n}\right)}:=|\mu|\left(\mathbb{R}^{n}\right), \mathrm{M}\left(\mathbb{R}^{n}, \mathbb{C}^{n}\right)$ is a Banach space.

Proof. (a) See [6]. (b) The space $\mathrm{M}\left(\mathbb{R}^{n}, \mathbb{C}^{n}\right)$ is isomorphic to $\mathrm{M}\left(\mathbb{R}^{n}, \mathbb{C}\right)^{n}$.

Since $\mathbb{C}^{n}$ has the Radon-Nikodým property, each $\eta$-continuous $\mu \in \mathrm{M}\left(\mathbb{R}^{n}, \mathbb{C}^{n}\right)$ has a Radon-Nikodým derivative with respect to a nonnegative Radon measure $\eta$. To be precise, for each $\mu \in \mathrm{M}\left(\mathbb{R}^{n}, \mathbb{C}^{n}\right)$ and $\eta \in \mathrm{M}\left(\mathbb{R}^{n},[0, \infty)\right)$ such that $\eta(\mathcal{O})=0$ implies $\mu(\mathcal{O})=0$ there exists a $g \in L^{1}\left(\mathbb{R}^{n}, \eta, \mathbb{C}^{n}\right)$ such that

$$
\mu(\mathcal{O})=\int_{\mathcal{O}} g \mathrm{~d} \eta, \quad \mathcal{O} \in \mathscr{B} .
$$

In particular, we have the representation

$$
\mu(\mathcal{O})=\int_{\mathcal{O}} \nu_{\mu} \mathrm{d}|\mu|, \quad \mathcal{O} \in \mathscr{B},
$$

with a $\nu \in L^{1}\left(\mathbb{R}^{n},|\mu|\right)$ such that $\left|\nu_{\mu}\right|(x)=1\left(x \in \mathbb{R}^{n}\right)$ (see [6]). Since each $\mu \in$ $\mathrm{M}\left(\mathbb{R}^{n}, \mathbb{C}^{n}\right)$ is defined on $\mathscr{B}$, the expression

$$
\mu\left\lfloor\psi(\mathcal{O}):=\int_{\mathcal{O}} \psi \nu_{\mu} \mathrm{d}|\mu|, \quad \mathcal{O} \in \mathscr{B},\right.
$$

is well-defined for every $\psi \in \mathrm{BC}\left(\mathbb{R}^{n}, \mathscr{L}\left(\mathbb{C}^{n}\right)\right)$. The proof of the following properties is straightforward.

Lemma 2.3. Let $\mu \in \mathrm{M}\left(\mathbb{R}^{n}, \mathbb{C}^{n}\right)$ and $\psi, \phi \in \mathrm{BC}\left(\mathbb{R}^{n}, \mathscr{L}\left(\mathbb{C}^{n}\right)\right)$ be given. Then we have

(i) $\mid \mu\lfloor\psi|\leq| \mu \mid\lfloor|\psi|$,

(ii) $\mu\left\lfloor\psi \in \mathrm{M}\left(\mathbb{R}^{n}, \mathbb{C}^{n}\right)\right.$,

(iii) $(\mu\lfloor\psi)\lfloor\phi=\mu\lfloor(\phi \psi)$.

In our applications to the Navier-Stokes equations we will frequently have $\psi=$ $\sigma_{P}$, where $\sigma_{P}(\xi)=I-\xi \xi^{T} /|\xi|^{2}$ denotes the symbol of the Helmholtz projection on $\mathbb{R}^{n}$. However, $\sigma_{P}$ is discontinuous at $\xi=0$. This motivates the introduction of

$$
\mathrm{M}_{0}\left(\mathbb{R}^{n}, C^{n}\right):=\left\{\mu \in \mathrm{M}\left(\mathbb{R}^{n}, C^{n}\right): \mu(\{0\})=0\right\},
$$

which is a closed subspace of $\mathrm{M}\left(\mathbb{R}^{n}, C^{n}\right)$. It is this space which will be used in our applications in the next section. Next, note that by the identification

$$
f \mapsto \lambda\left\lfloor f, \quad f \in L^{1}\left(\mathbb{R}^{n}, \mathbb{C}^{n}\right),\right.
$$


where $\lambda$ denotes the Lebesgue measure on $\mathbb{R}^{n}$, we see that every $f \in L^{1}\left(\mathbb{R}^{n}, \mathbb{C}^{n}\right)$ has a unique representant in $\mathrm{M}\left(\mathbb{R}^{n}, \mathbb{C}^{n}\right)$. On the other hand, the identification

$$
\mu \mapsto T_{\mu}, T_{\mu} f:=\mu\left\lfloor f, \quad f \in \mathcal{S}\left(\mathbb{R}^{n}, \mathbb{C}^{n}\right),\right.
$$

where $\mathcal{S}\left(\mathbb{R}^{n}, \mathbb{C}^{n}\right)$ denotes the Schwartz space of rapidly decreasing functions, shows that each $\mu \in \mathrm{M}\left(\mathbb{R}^{n}, \mathbb{C}^{n}\right)$ can be regarded as a tempered distribution. Altogether we have

$$
L^{1}\left(\mathbb{R}^{n}, \mathbb{C}^{n}\right) \hookrightarrow \mathrm{M}_{0}\left(\mathbb{R}^{n}, \mathbb{C}^{n}\right) \hookrightarrow \mathrm{M}\left(\mathbb{R}^{n}, \mathbb{C}^{n}\right) \hookrightarrow \mathcal{S}^{\prime}\left(\mathbb{R}^{n}, \mathbb{C}^{n}\right)
$$

Thus, the Fourier transform on $\mathrm{M}\left(\mathbb{R}^{n}, \mathbb{C}^{n}\right)$ is well-defined and we have $\widehat{\mu}(\xi)=$ $\mu\left\lfloor\varphi_{\xi}\left(\mathbb{R}^{n}\right)\right.$ with $\varphi_{\xi}(x)=(2 \pi)^{-n / 2} e^{-i x \cdot \xi}$. This allows for the introduction of the space

$$
\mathrm{FM}_{0}\left(\mathbb{R}^{n}, \mathbb{C}^{n}\right):=\left\{\widehat{\mu}: \mu \in \mathrm{M}_{0}\left(\mathbb{R}^{n}, \mathbb{C}^{n}\right)\right\},
$$

which we equip with the canonical norm $\|u\|_{\mathrm{FM}}:=\left\|\mathcal{F}^{-1} u\right\|_{\mathrm{M}}$. Observe that by definition we have $\|\mathcal{F} u\|_{\mathrm{M}}=\left\|\mathcal{F}^{-1} u\right\|_{\mathrm{M}}$. The space $\operatorname{FM}\left(\mathbb{R}^{n}, \mathbb{C}^{n}\right)$ is defined accordingly. Finally, we define the convolution of finite Radon measures as follows

$$
\begin{aligned}
& *: \mathrm{M}\left(\mathbb{R}^{n}, \mathbb{C}\right) \times \mathrm{M}\left(\mathbb{R}^{n}, \mathbb{C}^{n}\right) \rightarrow \mathrm{M}\left(\mathbb{R}^{n}, \mathbb{C}^{n}\right), \\
& \eta * \mu(\mathcal{O}):=\int_{\mathbb{R}^{n}} \eta(\mathcal{O}-x) \nu_{\mu}(x) \mathrm{d}|\mu|(x), \quad \mathcal{O} \in \mathscr{B} .
\end{aligned}
$$

The following properties are straightforward consequences of the definitions.

Lemma 2.4. We have

(i) $\mathcal{F}(\eta * \mu)=(2 \pi)^{n / 2} \widehat{\eta} \cdot \widehat{\mu}, \quad \eta \in \mathrm{M}\left(\mathbb{R}^{n}, \mathbb{C}\right), \mu \in \mathrm{M}\left(\mathbb{R}^{n}, \mathbb{C}^{n}\right)$,

(ii) $\|u v\|_{\mathrm{FM}\left(\mathbb{R}^{n}, \mathbb{C}^{n}\right)} \leq(2 \pi)^{-n / 2}\|u\|_{\mathrm{FM}\left(\mathbb{R}^{n}, \mathbb{C}\right)}\|v\|_{\mathrm{FM}\left(\mathbb{R}^{n}, \mathbb{C}^{n}\right)}$,

(iii) $\mathcal{F} L^{1}\left(\mathbb{R}^{n}, \mathbb{C}^{n}\right) \hookrightarrow \mathrm{FM}_{0}\left(\mathbb{R}^{n}, \mathbb{C}^{n}\right) \hookrightarrow \dot{B}_{\infty, 1}^{0}\left(\mathbb{R}^{n}, \mathbb{C}^{n}\right) \hookrightarrow \mathrm{BUC}\left(\mathbb{R}^{n}, \mathbb{C}^{n}\right) / \mathbb{C}^{n}$.

Here $\dot{B}_{\infty, 1}^{0}\left(\mathbb{R}^{n}, \mathbb{C}^{n}\right)$ denotes the homogeneous Besov space.

The next result, given in [10], is essential for the uniformness of our results in the Coriolis parameter $\Omega$. It is obtained as a consequence of Lemma 2.3.

Proposition 2.5. Suppose that $\sigma \in \mathrm{BC}\left(\mathbb{R}^{n} \backslash\{0\}, \mathbb{C}^{n \times n}\right)$. Then, we have

$$
\begin{aligned}
& O p(\sigma):=\mathcal{F}^{-1} \sigma \mathcal{F} \in \mathscr{L}\left(\mathrm{FM}_{0}\left(\mathbb{R}^{n}, \mathbb{C}^{n}\right)\right), \\
& \|O p(\sigma)\|_{\mathscr{L}\left(\mathrm{FM}_{0}\left(\mathbb{R}^{n}, \mathbb{C}^{n}\right)\right)}=\|\sigma\|_{L^{\infty}\left(\mathbb{R}^{n} \backslash\{0\}, \mathbb{C}^{n \times n}\right)} .
\end{aligned}
$$

If $\sigma$ is also continuous at the origin, then $O p(\sigma) \in \mathscr{L}\left(\mathrm{FM}\left(\mathbb{R}^{n}, \mathbb{C}^{n}\right)\right)$ with the corresponding equality for the operator norm. 
Let $\sigma_{P}(\xi)=I-\xi \xi^{T} /|\xi|^{2}$ be the symbol of the Helmholtz projection, where $P(\xi)$ is the symbol of the operator $P$ (projection on divergence free vector fields in $\mathbb{R}^{n}$ ). Next, set $S:=P J P$ with

$$
J=\left(\begin{array}{rrrrr}
0 & -1 & 0 & \cdots & 0 \\
1 & 0 & 0 & & \\
0 & 0 & 0 & & \vdots \\
\vdots & & & \ddots & \\
0 & & \cdots & & 0
\end{array}\right) .
$$

We call $S$ the Poincaré-Riesz operator and denote its symbol by $\sigma_{S}=\sigma_{P} J \sigma_{P}$. Note that for dimension $n=3$ the solenoidal part of the Corolis force then can be represented as $\Omega P \mathbf{e}_{3} \times u=\Omega S u$. Observe that $\sigma_{P}$ is orthogonal, $\sigma_{S}$ is skew-symmetric, and thus $\sigma\left(\mathrm{e}^{t S}\right)$ is unitary on $\mathbb{C}^{n}$. The symbol of this operator is expressed in terms of classical Riesz operators. We refer to [11] for additional information. As a consequence of Proposition 2.5 we therefore obtain the following estimates (see [10, Lemma 2.5, Lemma 2.9]).

Lemma 2.6. (i) The operators $P, S$, and $\mathrm{e}^{t S}$ are bounded on $\mathrm{FM}_{0}\left(\mathbb{R}^{n}, \mathbb{C}^{n}\right)$. In particular, we have

$$
\begin{gathered}
\|P f\|_{\mathrm{FM}} \leq\|f\|_{\mathrm{FM}} \quad\left(f \in \mathrm{FM}_{0}\left(\mathbb{R}^{n}, \mathbb{C}^{n}\right)\right), \\
\left\|\mathrm{e}^{t S} f\right\|_{\mathrm{FM}} \leq\|f\|_{\mathrm{FM}} \quad\left(t \in \mathbb{R}, f \in \mathrm{FM}_{0}\left(\mathbb{R}^{n}, \mathbb{C}^{n}\right)\right) .
\end{gathered}
$$

(ii) The family $\left(e^{t \nu \Delta}\right)_{t \geq 0}$ is a bounded holomorphic $C_{0}$-semigroup of contractions on $\mathrm{FM}_{0}\left(\mathbb{R}^{n}, \mathbb{C}^{n}\right)$ and on $\operatorname{FM}\left(\mathbb{R}^{n}, \mathbb{C}^{n}\right)$, i.e.,

$$
\left\|e^{t \nu \Delta} f\right\|_{\mathrm{FM}} \leq\|f\|_{\mathrm{FM}} \quad\left(f \in \mathrm{FM}\left(\mathbb{R}^{n}, \mathbb{C}^{n}\right), t>0\right) .
$$

Next we consider the convolution of vector-valued functions and measure-valued functions. Let $T>0, \mathscr{A}$ be a $\sigma$-algebra, $g: \mathbb{R}^{n} \times(0, T) \rightarrow \mathbb{C}$ be a function, and

$$
f:(0, T) \rightarrow \mathrm{M}_{0}\left(\mathbb{R}^{n}, \mathbb{C}^{n}\right), \quad t \mapsto f(t),
$$

be a measure-valued function. If well-defined, we set

$$
g \star f(t)=\int_{0}^{t} f(s)\lfloor g(t-s) \mathrm{d} s, \quad t \in(0, T) .
$$

Note that (2.2) later will play the role of the solution of a Cauchy problem given by the variation of constant formula with $g$ as the kernel of the solution operator. 
Lemma 2.7. For all $f \in L^{1}\left((0, T), \mathrm{M}_{0}\left(\mathbb{R}^{n}, \mathbb{C}^{n}\right)\right)$ and $g \in \mathrm{BC}\left(\left(\mathbb{R}^{n} \backslash\{0\}\right) \times(0, T)\right)$ the convolution $g \star f$ is well-defined and we have

$$
\|g \star f\|_{L^{2}\left((0, T), \mathrm{M}_{0}\right)} \leq\|g\|_{L^{\infty}\left(\mathbb{R}^{n}, L^{2}(0, T)\right)}\|f\|_{L^{1}\left((0, T), \mathrm{M}_{0}\right)} \quad(0<T \leq \infty) .
$$

Remark 2.8. At a first glance (2.3) might look like a Young type inequality. But, observe that for later purposes it is essential that on the right hand side we take the $L^{\infty}\left(L^{2}\right)$-norm of $g$ and not the $L^{2}\left(L^{\infty}\right)$-norm as usual in Young's inequality. The fact that always $\|\cdot\|_{L^{\infty}\left(R^{n}, L^{2}(0, T)\right)} \leq\|\cdot\|_{L^{2}\left((0, T), L^{\infty}\left(R^{n}\right)\right)}$ but the converse is not true in general shows that (2.3) is sharper than Young's inequality. Indeed, we will see that (2.3) gives an estimate for singular integral operators, which is not possible with the standard Young's inequality.

Proof. Since $g(s) \in \mathrm{BC}\left(\mathbb{R}^{n} \backslash\{0\}\right)$ and $f(s) \in \mathrm{M}_{0}\left(\mathbb{R}^{n}, \mathbb{C}^{n}\right)$ for almost all $s \in(0, T)$, by Lemma 2.3 we have $f(s)\left\lfloor g(t-s) \in \mathrm{M}_{0}\left(\mathbb{R}^{n}, \mathbb{C}^{n}\right)\right.$ and

$$
\begin{aligned}
\| f(s)\left\lfloor g(t-s) \|_{\mathrm{M}_{0}}\right. & =\mid f(s)\left\lfloor g(t-s) \mid\left(\mathbb{R}^{n}\right)\right. \\
& \leq\|g(t-s)\|_{L^{\infty}\left(\mathbb{R}^{n}\right)}\|f(s)\|_{\mathrm{M}_{0}} \quad(s \in(0, t)) .
\end{aligned}
$$

The assumptions on $f$ and $g$ imply that $\left(s \mapsto \| f(s)\left\lfloor g(t-s) \|_{\mathrm{M}_{0}}\right) \in L^{1}((0, t))\right.$. Hence, $g \star f(t)$ is well-defined as a Bochner integral. Next, set $\widetilde{g}(t):=\chi_{(0, T)}(t) g(t)$ and $\tilde{f}(t):=\chi_{(0, T)}(t) f(t)$, where $\chi_{E}$ denotes the indicator function of the set $E$. Then, $\widetilde{g} \in L^{\infty}\left(\mathbb{R}^{n} \times \mathbb{R}\right), \tilde{f} \in L^{1}\left(\mathbb{R}, \mathrm{M}_{0}\right)$ and we have

$$
g \star f(t)=\int_{\mathbb{R}} \widetilde{f}(s)\lfloor\widetilde{g}(t-s) \mathrm{d} s .
$$

This implies that

$$
\|g \star f(t)\|_{\mathrm{M}_{0}} \leq \int_{\mathbb{R}} \int_{\mathbb{R}^{n}}|\widetilde{g}(\xi, t-s)| \mathrm{d}|\widetilde{f}(s)|(\xi) \mathrm{d} s, \quad t \in(0, T) .
$$

By the fact that

$$
\int_{0}^{T}|\widetilde{g}(\xi, t-s)|^{2} \mathrm{~d} t \leq \int_{0}^{T}|g(\xi, r)|^{2} \mathrm{~d} r=\|g(\xi, \cdot)\|_{L^{2}(0, T)}^{2}, \quad s \in(0, T),
$$

taking the $L^{2}$-norm on both sides of (2.4) results in

$$
\begin{aligned}
\|g \star f\|_{L^{2}\left((0, T), \mathrm{M}_{0}\right)} & \leq \int_{\mathbb{R}} \int_{\mathbb{R}^{n}}\|g(\xi, \cdot)\|_{L^{2}(0, T)} \mathrm{d}|\tilde{f}(s)|(\xi) \mathrm{d} s \\
& \leq\|g\|_{L^{\infty}\left(\mathbb{R}^{n}, L^{2}(0, T)\right)}\|f\|_{L^{1}\left((0, T), \mathrm{M}_{0}\right)} \quad(0<T \leq \infty) .
\end{aligned}
$$


The rigorous definition of the critical space for the Navier-Stokes equations in the Radon measure setting is

$$
\mathrm{FM}_{0}^{-1}\left(\mathbb{R}^{n}, \mathbb{C}^{n}\right):=\left\{f \in \mathcal{S}\left(\mathbb{R}^{n}, \mathbb{C}^{n}\right): \widehat{f}\left\lfloor(1 /|\cdot|) \in \mathrm{M}_{0}\left(\mathbb{R}^{n}, \mathbb{C}^{n}\right)\right\}\right.
$$

with canonical norm $\|f\|_{-1}=\|f\|_{\mathrm{FM}_{0}^{-1}}=\| \widehat{f}\left\lfloor(1 /|\cdot|) \|_{\mathrm{M}_{0}}\right.$.

Lemma 2.9. The following assertions on the space $\mathrm{FM}_{0}^{-1}\left(\mathbb{R}^{n}, \mathbb{C}^{n}\right)$ hold:

(i) $\mathrm{FM}_{0}^{-1}\left(\mathbb{R}^{n}, \mathbb{C}^{n}\right)=\operatorname{div} \mathrm{FM}_{0}\left(\mathbb{R}^{n}, \mathbb{C}^{n}\right)^{n}$, where

$$
\operatorname{div} \mathrm{FM}_{0}\left(\mathbb{R}^{n}, \mathbb{C}^{n}\right)^{n}=\left\{f=\sum_{j=1}^{n} \partial_{j} g_{j}: g_{j} \in \mathrm{FM}_{0}\left(\mathbb{R}^{n}, \mathbb{C}^{n}\right)(j=1, \ldots, n)\right\}
$$

(ii) $\mathrm{FM}_{0}^{-1}\left(\mathbb{R}^{n}, \mathbb{C}^{n}\right)$ is a Banach space.

(iii) The assertions of Proposition 2.5 and Lemma 2.6 remain valid if $\mathrm{FM}_{0}$ is replaced by $\mathrm{FM}_{0}^{-1}$.

Proof. (i) Note that according to Proposition 2.5, $\xi \mapsto i \xi /|\xi|$ is the symbol of a bounded operator on $\operatorname{FM}_{0}\left(\mathbb{R}^{n}, \mathbb{C}^{n}\right)$. Thus, for $f=\sum_{j=1}^{n} \partial_{j} g_{j}$ we have $f \in$ $\mathcal{S}^{\prime}\left(\mathbb{R}^{n}, \mathbb{C}^{n}\right)$ and

$$
\frac{\widehat{f}}{|\xi|}=\sum_{j=1}^{n} \frac{i \xi}{|\xi|} \widehat{g}_{j} \in \mathrm{M}_{0}\left(\mathbb{R}^{n}, \mathbb{C}^{n}\right)
$$

if $g_{j} \in \mathrm{FM}_{0}\left(\mathbb{R}^{n}, \mathbb{C}^{n}\right)$ for $j=1, \ldots, n$. This shows " $\supseteq$ ". In order to see the converse inclusion, pick $f \in \mathrm{FM}_{0}^{-1}\left(\mathbb{R}^{n}, \mathbb{C}^{n}\right)$. Then, write $\widehat{f}$ as

$$
\widehat{f}=-\sum_{j=1}^{n} \frac{\left(i \xi_{j}\right)^{2}}{|\xi|^{2}} \widehat{f}=\sum_{j=1}^{n} i \xi_{j}\left(-\frac{i \xi_{j}}{|\xi|^{2}} \widehat{f}\right) .
$$

By assumption we have $\widehat{g}_{j}:=-\frac{i \xi}{|\xi|^{2}} \widehat{f} \in \mathrm{M}_{0}\left(\mathbb{R}^{n}, \mathbb{C}^{n}\right)$, which proves the claim.

(ii) The operator $M:=\mathcal{F}^{-1}[|\xi|] \mathcal{F}$ is injective from $\mathrm{FM}_{0}\left(\mathbb{R}^{n}, \mathbb{C}^{n}\right)$ to $\mathcal{S}^{\prime}\left(\mathbb{R}^{n}, \mathbb{C}^{n}\right)$. Hence, $M: \mathrm{FM}_{0}\left(\mathbb{R}^{n}, \mathbb{C}^{n}\right) \rightarrow \mathrm{FM}_{0}^{-1}\left(\mathbb{R}^{n}, \mathbb{C}^{n}\right)$ is an isomorphism. The assertion therefore follows, since $\mathrm{FM}_{0}\left(\mathbb{R}^{n}, \mathbb{C}^{n}\right)$ is a Banach space.

(iii) This is obvious by the definition and since $1 /|\xi|$ commutes with all appearing symbols.

Observe that by Lemma 2.6(i) we easily obtain the Helmholtz decomposition

$$
\mathrm{FM}_{0}\left(\mathbb{R}^{n}, \mathbb{C}^{n}\right)=\mathrm{FM}_{0, \sigma}\left(\mathbb{R}^{n}, \mathbb{C}^{n}\right) \oplus \mathrm{G}_{F M}
$$


where

$$
\mathrm{FM}_{0, \sigma}\left(\mathbb{R}^{n}, \mathbb{C}^{n}\right):=P \mathrm{FM}_{0}\left(\mathbb{R}^{n}, \mathbb{C}^{n}\right)=\left\{u \in \mathrm{FM}_{0}\left(\mathbb{R}^{n}, \mathbb{C}^{n}\right): \operatorname{div} u=0\right\}
$$

and $\mathrm{G}_{\mathrm{FM}}=\left\{\nabla p: p \in \mathcal{S}^{\prime}\left(\mathbb{R}^{n}, \mathbb{C}^{n}\right), \nabla p \in \mathrm{FM}_{0}\left(\mathbb{R}^{n}, \mathbb{C}^{n}\right)\right\}$.

Now we are in position to prove the key estimates for the semiroup $\left(e^{-t A}\right)_{t \geq 0}$ generated by the Stokes-Poincaré-Riesz operator given by

$$
A=-\nu \Delta+\Omega S
$$

and defined in $\mathrm{FM}_{0, \sigma}\left(\mathbb{R}^{n}, \mathbb{C}^{n}\right)$. Lemma 2.6(i) and (ii) immediately imply that $\left(e^{-t A}\right)_{t \geq 0}$ is a holomorphic $C_{0}$-semigroup of contractions on $\mathrm{FM}_{0, \sigma}\left(\mathbb{R}^{n}, \mathbb{C}^{n}\right)$. For $f$ and $u_{0}$ suitably chosen,

$$
u(t)=e^{-t A} u_{0}+\int_{0}^{t} e^{-(t-s) A} f(s) \mathrm{d} s, \quad t>0,
$$

therefore represents the unique solution of the Cauchy problem

$$
\left\{\begin{aligned}
u_{t}+A u & =f, \quad t>0 \\
u(0) & =u_{0}
\end{aligned}\right.
$$

Thanks to Lemma 2.9, exactly the same statements on $P$ and $\left(e^{-t A}\right)_{t \geq 0}$ hold for the space $\mathrm{FM}_{0}^{-1}\left(\mathbb{R}^{n}, \mathbb{C}^{n}\right)$. In particular, the space $\mathrm{FM}_{0, \sigma}^{-1}\left(\mathbb{R}^{n}, \mathbb{C}^{n}\right)$ is defined analogously as the space $\mathrm{FM}_{0, \sigma}\left(\mathbb{R}^{n}, \mathbb{C}^{n}\right)$. The mentioned key estimates read as follows.

Lemma 2.10. Let $u_{0} \in \mathrm{FM}_{0, \sigma}^{-1}\left(\mathbb{R}^{n}, \mathbb{C}^{n}\right), f \in L^{1}\left((0, T), \mathrm{FM}_{0, \sigma}\left(\mathbb{R}^{n}, \mathbb{C}^{n}\right)\right)$, and $u$ be the solution of (2.6) given by (2.5).

(i) If $f \equiv 0$, we have

$$
\|u\|_{L^{2}\left((0, T), \mathrm{FM}_{0}\right)} \leq \frac{1}{\sqrt{2 \nu}}\left\|u_{0}\right\|_{-1} \quad(0<T \leq \infty) .
$$

(ii) If $u_{0} \equiv 0$, we have

$$
\left\|(-\Delta)^{1 / 2} u\right\|_{L^{2}\left((0, T), \mathrm{FM}_{0}\right)} \leq \frac{1}{\sqrt{2 \nu}}\|f\|_{L^{1}\left((0, T), \mathrm{FM}_{0}\right)} \quad(0<T \leq \infty) .
$$

Proof. (i) From Lemma 2.6(i) we infer that

$$
\|u(t)\|_{\mathrm{FM}_{0}} \leq\left\|e^{-\nu t \Delta} u_{0}\right\|_{\mathrm{FM}_{0}} \leq \int_{\mathbb{R}^{n}} e^{-\nu t|\xi|^{2}} \mathrm{~d}\left|\widehat{u}_{0}\right|(\xi) .
$$

It is easily calculated that

$$
\left\|e^{-\nu(\cdot)|\xi|^{2}}\right\|_{L^{2}(0, T)} \leq \frac{1}{|\xi| \sqrt{2 \nu}} \quad\left(0<T \leq \infty, \xi \in \mathbb{R}^{n} \backslash\{0\}\right) .
$$


Regarding $e^{-\nu(\cdot)|\xi|^{2}}$ as an $L^{2}(0, T)$-valued function and the right hand side of (2.7) as a $\left(L^{2}\right.$-valued) Bochner integral, this implies that

$$
\|u\|_{L^{2}\left((0, T), \mathrm{FM}_{0}\right)} \leq \int_{\mathbb{R}^{n}}\left\|e^{-\nu(\cdot)|\xi|^{2}}\right\|_{L^{2}(0, T)} \mathrm{d}\left|\widehat{u}_{0}\right|(\xi) \leq \frac{1}{\sqrt{2 \nu}}\left\|u_{0}\right\|_{-1} \quad(0<T \leq \infty) .
$$

(ii) We have

$$
\mathcal{F}(-\Delta)^{1 / 2} u(t)=\int_{0}^{t} \widehat{f}(s)\lfloor g(\cdot, t-s) \mathrm{d} s
$$

with $g(\xi, s)=|\xi| e^{-(t-s)\left(\nu|\xi|^{2}+\Omega \sigma_{S}(\xi)\right)}$. Lemma 2.7 now implies

$$
\left\|(-\Delta)^{1 / 2} u\right\|_{L^{2}\left((0, T), \mathrm{FM}_{0}\right)} \leq\|g\|_{L^{\infty}\left(\mathbb{R}^{n}, L^{2}(0, T)\right)}\|f\|_{L^{1}\left((0, T), \mathrm{FM}_{0}\right)} .
$$

By virtue of

$$
\|g\|_{L^{\infty}\left(\mathbb{R}^{n}, L^{2}(0, T)\right)}=\sup _{\xi \in \mathbb{R}^{n}}\left(\int_{0}^{T}|\xi|^{2} e^{-2 t\left(\nu|\xi|^{2}-\Omega \sigma_{S}(\xi)\right)} \mathrm{d} t\right)^{1 / 2} \leq \frac{1}{\sqrt{2 \nu}} \quad(0<T \leq \infty),
$$

where we used the skew-symmetry of $\sigma_{S}(\xi)$ in the first inequality, we obtain the assertion.

\section{Proof of the main results}

We show that

$$
H u(t):=e^{-t A} u_{0}+\int_{0}^{t}(-\Delta)^{1 / 2} e^{-(t-s) A} P(u(s) \cdot \nabla)(-\Delta)^{-1 / 2} u(s) \mathrm{d} s
$$

is a contraction on

$$
B_{u_{0}}:=\left\{u \in L^{2}\left(\mathbb{R}_{+}, \mathrm{FM}_{0, \sigma}\left(\mathbb{R}^{n}, \mathbb{C}^{n}\right)\right):\|u\|_{L^{2}\left(\mathbb{R}_{+}, \mathrm{FM}_{0}\right)} \leq \sqrt{2 / \nu}\left\|u_{0}\right\|_{-1}\right\} .
$$

Indeed, Lemma 2.10(i) and (ii) give us

$$
\|H u\|_{L^{2}\left(\mathbb{R}_{+}, \mathrm{FM}_{0}\right)} \leq \frac{1}{\sqrt{2 \nu}}\left(\left\|u_{0}\right\|_{-1}+\left\|P(u \cdot \nabla)(-\Delta)^{-1 / 2} u\right\|_{L^{1}\left(\mathbb{R}_{+}, \mathrm{FM}_{0}\right)}\right) .
$$

By the boundedness of $P$ and $\partial_{j}(-\Delta)^{-1 / 2}$ on $\mathrm{FM}_{0}\left(\mathbb{R}^{n}, \mathbb{C}^{n}\right)$ and Lemma 2.4(ii) we further obtain

$$
\left\|P(u \cdot \nabla)(-\Delta)^{-1 / 2} u\right\|_{L^{1}\left(\mathbb{R}_{+}, \mathrm{FM}_{0}\right)} \leq \frac{1}{(2 \pi)^{n / 2}} \sum_{j=1}^{n}\left\|u^{j}\right\|_{L^{2}\left(\mathbb{R}_{+}, \mathrm{FM}_{0}\right)}\|u\|_{L^{2}\left(\mathbb{R}_{+}, \mathrm{FM}_{0}\right)} .
$$


The fact that $\max _{1 \leq j \leq n} a_{j} \leq n^{-1 / 2}\left(\sum_{j=1}^{n} a_{j}\right)^{1 / 2}$ for $a_{j} \geq 0, j=1, \ldots, n$, yields

$$
\sum_{j=1}^{n}\left\|u^{j}(t)\right\|_{\mathrm{FM}_{0}} \leq n \sup _{\Pi\left(\mathbb{R}^{n}\right)} \sum_{E \in \Pi\left(\mathbb{R}^{n}\right)} \max _{1 \leq j \leq n}\left|\widehat{u}^{j}(t, E)\right| \leq \sqrt{n}\|u(t)\|_{\mathrm{FM}_{0}} .
$$

Consequently,

$$
\left\|P(u \cdot \nabla)(-\Delta)^{-1 / 2} u\right\|_{L^{1}\left(\mathbb{R}_{+}, \mathrm{FM}_{0}\right)} \leq \frac{\sqrt{n}}{(2 \pi)^{n / 2}}\|u\|_{L^{2}\left(\mathbb{R}_{+}, \mathrm{FM}_{0}\right)}^{2} .
$$

Inserting this into (3.1) and taking into account that $u \in B_{u_{0}}$ results in

$$
\begin{aligned}
\|H u\|_{L^{2}\left(\mathbb{R}_{+}, \mathrm{FM}_{0}\right)} & \leq \frac{1}{\sqrt{2 \nu}}\left(\left\|u_{0}\right\|_{-1}+\frac{\sqrt{n}}{(2 \pi)^{n / 2}}\|u\|_{L^{2}\left(\mathbb{R}_{+}, \mathrm{FM}_{0}\right)}^{2}\right) \\
& \leq \frac{1}{\sqrt{2 \nu}}\left\|u_{0}\right\|_{-1}\left(1+\frac{2 \sqrt{n}}{\nu(2 \pi)^{n / 2}}\left\|u_{0}\right\|_{-1}\right) .
\end{aligned}
$$

This shows that $\|H u\|_{L^{2}\left(\mathbb{R}_{+}, \mathrm{FM}_{0}\right)} \leq \sqrt{2 / \nu}\left\|u_{0}\right\|_{-1}$, if $\left\|u_{0}\right\|_{-1} \leq \nu(2 \pi)^{n / 2} / 2 \sqrt{n}$. Furthermore, for $u, v \in B_{u_{0}}$ we write

$$
(u \cdot \nabla) u-(v \cdot \nabla) v=((u-v) \cdot \nabla) u+(v \cdot \nabla)(u-v) .
$$

Then, we obtain completely analogous as above that

$$
\begin{aligned}
\|H u-H v\|_{L^{2}\left(\mathbb{R}_{+}, \mathrm{FM}_{0}\right) \leq} \leq & \frac{1}{\sqrt{2 \nu}} \frac{\sqrt{n}}{(2 \pi)^{n / 2}}\left(\|u\|_{L^{2}\left(\mathbb{R}_{+}, \mathrm{FM}_{0}\right)}^{2}+\|v\|_{L^{2}\left(\mathbb{R}_{+}, \mathrm{FM}_{0}\right)}^{2}\right) \\
& \cdot\|u-v\|_{L^{2}\left(\mathbb{R}_{+}, \mathrm{FM}_{0}\right)} \\
\leq & \frac{2 \sqrt{n}}{\nu(2 \pi)^{n / 2}}\left\|u_{0}\right\|_{-1}\|u-v\|_{L^{2}\left(\mathbb{R}_{+}, \mathrm{FM}_{0}\right)} .
\end{aligned}
$$

Thus, $H$ is a contraction, if $\left\|u_{0}\right\|_{-1}<\nu(2 \pi)^{n / 2} / 2 \sqrt{n}$ which is exactly condition (1.2). This completes the proof of Theorem 1.1.

Next we prove Theorem 1.3. The boundedness (1.3) in $L^{2}([0, T] ; \mathrm{FM})(0<T \leq \infty)$ can be seen as in Lemma 2.10 by a similar estimate for $\mathcal{F}\left(\nabla_{j} u\right)=i \xi_{j} \widehat{u}$,

$$
\begin{aligned}
\|\nabla u\|_{L^{2}\left((0, T), \mathrm{FM}_{0}\right)} & \leq \int_{\mathbb{R}^{n}}\left\|i \xi_{j} e^{-\nu(\cdot)|\xi|^{2}}\right\|_{L^{2}(0, T)} \mathrm{d}\left|\widehat{u}_{0}\right|(\xi) \\
& \leq \int_{\mathbb{R}^{n}} \frac{|\xi|}{|\xi| \sqrt{2 \nu}} \mathrm{d}\left|\widehat{u}_{0}\right|(\xi) \leq \frac{1}{\sqrt{2 \nu}}\left\|u_{0}\right\|_{\mathrm{FM}_{0}} \quad(0<T \leq \infty)
\end{aligned}
$$

since $\left\|i \xi_{j} e^{-\nu(\cdot)|\xi|^{2}}\right\|_{L^{2}(0, T)} \leq|\xi| /(|\xi| \sqrt{2 \nu})$. (note that now $u_{0}$ belongs to not only $\mathrm{FM}_{0}^{-1}$ but also $\mathrm{FM}_{0}$ ). Proofs of (2) and (3) of Theorem 1.3 are very similar to the 
proof of Theorem 1 ii) in [9], where corresponding results in the space of uniformly continuous functions BUC are proved. Adapting the Stokes-Poincaré-Riesz operator $A=-\nu \Delta+\Omega S$ and $f(s)=P \operatorname{div} u(s) u(s)^{T}$ to $(2.5)$ we have

$$
u(t)=\mathrm{e}^{-A t} u_{0}-\int_{0}^{t} P \operatorname{div} \mathrm{e}^{-A(t-s)} u(s) u(s)^{T} \mathrm{~d} s \quad \text { for } \quad t>0,
$$

Taking the FM-norm on both hand sides of this integral equation, the exponential term of the Coriolis operator $\Omega \mathbf{S}$ is uniformly bounded in our space by virtue of the uniform estimate

$$
\|\exp (\Omega S t) f\|_{\mathrm{FM}} \leq\|f\|_{\mathrm{FM}}
$$

(Lemma 2.6). Then the proof proceeds in the same way as for the integral equation corresponding to the Navier-Stokes equations without the Coriolis term

$$
u(t)=\mathrm{e}^{\nu t \Delta} u_{0}-\int_{0}^{t} P \operatorname{div} \mathrm{e}^{\nu(t-s) \Delta} u(s) v(s)^{T} \mathrm{~d} s \quad \text { for } \quad t>0,
$$

for which we can repeat the proof of regularity as in [9], replacing BUC by FM.

Theorem 1.4 is also proven as in [9]. However, we note that we cannot conclude that the mild solution (with the pressure (1.6)) obtained in the theorems 1.1 and 1.3 is a global-in-time classical solution. This is because the proof for regularity (Theorem 1.3 ) is based on estimates for the solution $u(t)$ of the integral equation (3.2). Even by taking spacial derivatives in order to estimate $\|\nabla u(t)\|_{\mathrm{FM}}$, or by taking time difference $\|u(t+h)-u(t)\|_{\mathrm{FM}}$ on both hand sides of the above integral equation, there seems to be no chance to calculate the square of the FM-norm, $\|\nabla u(t)\|_{\mathrm{FM}}^{2}$ nor $\|u(t+h)-u(t)\|_{\mathrm{FM}}^{2}$. However, our global-in-time estimates (Lemma 2.10) hold only in the $L^{2}$-space in time - $L^{2}((0, \infty) ; \mathrm{FM})$. Hence, the mild solution can be proved to be classical at most locally in time $T_{0}<\infty$.

\section{Acknowledgements.}

The work of the author Y.G. is partly supported by the Grant-in-Aid for Scientific Research, No. 18204011, the Japan Society for the Promotion of Science (JSPS). The work of the author K.I. was done when he was a part-time researcher in Automatic Control Laboratory at Musashi Institute of Technology sponsored by "Study for industrial use of strategic intelligence robots" (Professor Ben T. Nohara), subsidies for the promotion of private schools (the Ministry of Education, Culture, Sports, Science and Technology). Its hospitality is gratefully acknowledged. The work of the author A.M. is sponsored by AFOSR contract FA9550-08-1-0055. Also, it was done when he visited the University of Tokyo, the Center of Excellence (COE) 
program. Its hospitality is gratefully acknowledged. The work of the author J.S. is partially supported by the 'Ausschuß für Forschungsfragen' at the University of Konstanz.

\section{References}

[1] A. Babin, A. Mahalov, and B. Nicolaenko (1999), Global Regularity of the 3D Rotating Navier-Stokes Equations for Resonant Domains, Indiana University Mathematics Journal, 48, No. 3, p. 1133-1176.

[2] A. Babin, A. Mahalov, and B. Nicolaenko (2001), 3D Navier-Stokes and Euler equations with initial data characterized by uniformly large vorticity, Indiana Univ. Math. J., 50, 1-35.

[3] M. Cannone (2004), Harmonic Analysis Tools for Solving the Incompressible Navier-Stokes equations, Handbook of Mathematical Fluid Dynamics, Volume III, Chapter 3, 161-244.

[4] J.-Y. Chemin, B. Desjardins, I. Gallagher, and E. Grenier (2006), Mathematical geophysics, An introduction to rotating fluids and the Navier-Stokes equations, The Clarendon Press, Oxford.

[5] P. Constantin and C. Foias (1988), Navier-Stokes Equations, Chicago Lectures in Mathematics, The University of Chicago Press.

[6] J. Diestel and J. J. Uhl, Jr. (1977), Vector Measures, Mathematical Surveys Nr. 15, American Mathematical Society, Providence, Rhode Island.

[7] O. Dragicevic, S. Petermichl and A. Volberg (2006), A rotation method which gives linear $L^{p}$ estimates for powers of the Ahlfors-Beurling operator, J. Math. Pures Appl., 9, no.6, 86, 492-509.

[8] C. Foias, O. Manley, R. Rosa and R. Temam (2003), Navier-Stokes Equations and Turbulence, Cambridge University Press.

[9] Y. Giga, K. Inui and S. Matsui (1999), On the Cauchy problem for the NavierStokes equations with nondecaying initial data, Quaderni di Matematika, 4, p. 28-68.

[10] Y. Giga, K. Inui, A. Mahalov and S. Matsui (2005), Uniform local solvability for the Navier-Stokes equations with the Coriolis force, Methods and Applications of Analysis, 12, p. 381-394.

[11] Y. Giga, K. Inui, A. Mahalov and S. Matsui (2006), Navier-Stokes Equations in a Rotating Frame with Initial Data Nondecreasing at Infinity, Hokkaido Mathematical Journal, 35, No. 2, p. 321-364. 
[12] Y. Giga, K. Inui, A. Mahalov and J. Saal (2007), Global solvabiliy of the NavierStokes equations in spaces based on sum-closed frequency sets, Advances in Differential Equations, Vol. 12, Num. 7, p.721-736.

[13] Y. Giga, S. Matsui and O. Sawada (2001), Global existence of two dimensional Navier-Stokes flow with nondecaying initial velocity, J. Math. Fluid Mech.,3, 302-315 .

[14] H. Koch and D. Tataru (2001), Well-posedness for the Navier-Stokes equations, Advances in Math., 157, 22-35.

[15] A. Mahalov and B. Nicolaenko (2003), Global solubility of the threedimensional Navier-Stokes equations with uniformly large vorticity, Russ. Math. Surveys, 58, No. 2, 287-318.

[16] Y.Le Jan and A.-S. Sznitman (1997), Stochastic cascades and 3-dimensional Navier-Stokes equations, Probab. Theory Related Fields 109, 343-366.

[17] A.S. Monin and A.M. Yaglom (1971), Statistical Fluid Mechanics: Mechanics of Turbulence, Volume 1 and 2, MIT Press, Cambridge, Mass.

[18] M.J. Vishik and A.V. Fursikov (1988), Mathematical Problems of Statistical Hydromechanics, Kluwer Academic Publisher, Dordrecht, Netherlands, Boston. 